\title{
Arritmias en pacientes asintomáticos
}

\author{
Arrhythmia in asymptomatic patients.
}

\author{
Rodrigo Isa \\ Cardiólogo Electrofisiólogo \\ Jefe Unidad Coronaria clínica Redsalud Vitacura \\ Jefe de Cardiología clínica Redsalud Vitacura \\ Jefe unidad de arritmias Hospital el Carmen de Maipú
}

Señor Editor:

En el reciente número de Europace (2019; 0: 1-32), se publicó el documento de consenso de la asociación europea del ritmo cardiaco (EHRA) sobre el manejo de arritmias asintomáticas. Este documento fue realizado con la colaboración de la asociación de falla cardiaca (HFA), la Sociedad Norteamericana del Ritmo Cardiaco (HRS), la Sociedad del Ritmo Cardiaco de Asia Pacífico (APHRS), la Sociedad de Arritmias Cardiacas de Sudáfrica (CASSA), y la recientemente formada Sociedad Latinoamericana del Ritmo Cardiaco (LAHRS), en la cual participé como representante y coautor de esta guía clínica1.

Las arritmias asintomáticas corresponden a un capítulo muy poco abordado en la literatura médica especializada, y esto se debe a la falta de estudios clínicos específicamente diseñados para tratar este tema. Los pacientes que padecen algún tipo de arritmia y se encuentran "asintomáticos" son frecuentes en la práctica clínica y, generalmente, son diagnosticados incidentalmente en un control rutinario de salud, en una consulta por un problema no relacionado o cuando estamos en etapas avanzadas de la enfermedad.

Existen dos aristas muy importantes que los médicos debemos reflexionar respecto a este tópico: la primera, ¿se trata de pacientes "realmente asintomáticos"? o ¿es que la lenta instalación de la enfermedad produce una especie de acostumbramiento y efectivamente el paciente aprende constantemente a convivir con ella?. Para aclarar esto es fundamental la "pericia médica"; realizar una buena historia clínica y utilizar las pruebas complementarias necesarias. Ejemplo de esto son los pacientes que 
tienen una enfermedad del nodo sinusal, refieren no tener un síntoma específico y se han acostumbrado a vivir "a media máquina“, o los muchos pacientes que viven en fibrilación auricular y no notan la progresiva disminución de su clase funcional hasta etapas avanzadas de la enfermedad. Lo segundo, ¿los pacientes asintomáticos tiene mejor pronóstico que los pacientes sintomáticos? o en otras palabras, ¿los médicos estamos encomendados sólo a aliviar síntomas o a intentar tratar pacientes y cambiar el curso y pronóstico de sus enfermedades? Ejemplo de esto podrían ser pacientes con fibrilación auricular que, independiente, de sus síntomas, están expuestos al mismo riesgo cardioembólico y de desarrollo de insuficiencia cardiaca que los pacientes sintomáticos y que, intervenciones precoces en su evolución, podrían mejorar el curso de la enfermedad. Lo mismo podemos comentar sobre algunas arritmias ventriculares o condi- ciones pro-arritmogénicas en pacientes asintomáticos en que nuestro diagnóstico oportuno, seguido de una correcta toma de decisión, permitirán evitar severas consecuencias, como la muerte súbita de origen cardiaco.

Este documento, Management of asymptomatic arrhythmias: a European HeartRhythmAssociation (EHRA) consensus document, endorsedby the Heart Failure Association (HFA), Heart RhythmSociety (HRS), Asia Pacific Heart Rhythm Society (APHRS), Cardiac Arrhythmia Society of Southern Africa (CASSA), and LatinAmerica Heart RhythmSociety(LAHRS), revisa la evidencia, cuando está disponible, e intenta alcanzar un consenso, cuando la evidencia es insuficiente o en conflicto. Sin lugar a dudas es el inicio de un tema en permanente cambio y transformación del conocimiento, que además incorpora como elemento fundamental, un apartado sobre las perspectivas del paciente.

\section{Referencias}

1. ARNAR D, MAIRESSE G, BORIANI G, CALKINS H, CHIN A, COATS A, Management of asymptomatic arrhythmias: a European Heart RhythmAssociation (EHRA) consensus document, endorsed by the Heart Failure Association (HFA), Heart
Rhythm Society (HRS), Asia Pacific Heart Rhythm Society (APHRS), Cardiac Arrhythmia Society of Southern Africa (CASSA), and LatinAmerica Heart RhythmSociety (LAHRS). Europace 2019; 0: 1-32 